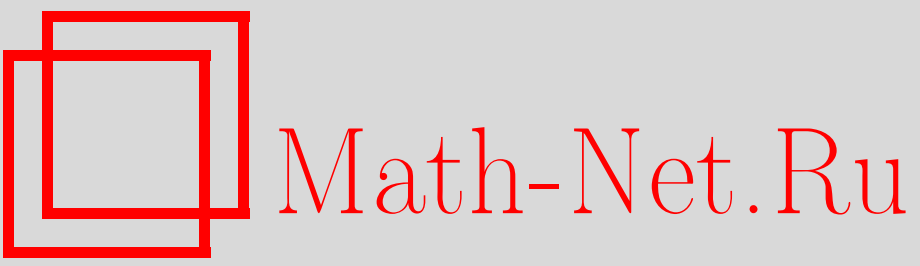

В. М. Бухштабер, Э. Г. Рис, Приложения фробениусовых $n$-гомоморфизмов, УМH, 2002, том 57, выпуск 1, 149-150

DOI: https://doi.org/10.4213/rm479

Использование Общероссийского математического портала Math-Net.Ru подразумевает, что вы прочитали и согласны с пользовательским соглашением

http://www.mathnet.ru/rus/agreement

Параметры загрузки:

IP: 54.197 .217 .227

26 апреля 2023 г., 16:15:15 


\title{
ПРИЛОЖЕНИЯ ФРОБЕНИУСОВЫХ $n$-ГОМОМОРФИЗМОВ
}

\author{
В. М. БУХШТАБЕР, Э.Г. РИС
}

Для каждого линейного отображения алгебр $f: A \rightarrow B$ определено полилинейное отображение $\Phi_{k}(f): A^{k} \rightarrow B$ при помощи рекурсии $\Phi$ робениуса $\left(\Phi_{1}=f\right)$ :

$$
\Phi_{k}(f)\left(a_{1}, \ldots, a_{k}\right)=\Phi_{1}\left(a_{1}\right) \Phi_{k-1}(f)\left(a_{2}, \ldots, a_{k}\right)-\sum_{j=2}^{k} \Phi_{k-1}(f)\left(a_{2}, \ldots, a_{1} a_{j}, \ldots, a_{k}\right) .
$$

ОПредЕЛЕниЕ [1]. Пусть $A$ и $B$ - алгебры с единицей. Отображение $f$ назьвается фробениусовьм $n$-гомоморфизмом, если $f(1)=n$ и $\Phi_{n+1}(f) \equiv 0$.

Результаты настоящей работы опираются на следующий наш результат [2]: линейное отображение $f: \mathbb{C}\left[u_{1}, \ldots, u_{m}\right] \rightarrow \mathbb{C}$ является фробениусовым $n$-гомоморфизмом тогда и только тогда, когда существует такой набор точек $X=\left[\mathbf{x}_{1}, \mathbf{x}_{2}, \ldots, \mathbf{x}_{n}\right]$ из $\mathbb{C}^{m}$, что $f(p)=p\left(\mathbf{x}_{1}\right)+\cdots+p\left(\mathbf{x}_{n}\right)$.

1. Для данных $m$ и $n$ рассмотрим отображение $g: \mathbb{C}^{m} \rightarrow \operatorname{Sym}^{n}(\mathbb{C})$. Пусть $s_{k}: \operatorname{Sym}^{n}(\mathbb{C}) \rightarrow \mathbb{C}$ - отображение, заданное $k$-м полиномом Ньютона $s_{k}\left[b_{1}, b_{2}, \ldots, b_{n}\right]=b_{1}^{k}+b_{2}^{k}+\cdots+b_{n}^{k}$. Обозначим через $\mathscr{N}_{m}$ полурешетку $\mathbb{Z}_{\geqslant 0}^{m}$. Для $\omega=\left(i_{1}, i_{2}, \ldots, i_{m}\right) \in \mathcal{N}_{m}$ положим $\mathbf{z}^{\omega}=z_{1}^{i_{1}} z_{2}^{i_{2}} \cdots z_{m}^{i_{m}}$ и $|\omega|=i_{1}+i_{2}+\cdots+i_{m}$.

Свойство 1. Композиция $s_{k} \circ g: \mathbb{C}^{m} \rightarrow \mathbb{C}$ задается формой $\sum_{|\omega|=k}\left(\begin{array}{l}k \\ \omega\end{array}\right) \alpha_{\omega} \mathbf{z}^{\omega}$ степени $k$ для всех $k=1,2, \ldots$, где $\left(\begin{array}{c}k \\ \omega\end{array}\right)=\frac{k !}{i_{1} ! i_{2} ! \cdots i_{m} !}$

Таким образом, если $g$ обладает свойством 1 , то формула $\Phi(\omega)=\alpha_{\omega}$ определяет отображение $\Phi: \mathscr{N}_{m} \rightarrow \mathbb{C}$.

Для $\omega=0$ положим $\Phi(0)=n$, и, задавая $\Phi_{1}=\Phi$, введем отображения $\Phi_{\ell}: \mathfrak{N}_{m}^{\ell} \rightarrow \mathbb{C}$ для $\ell>1$ при помощи следующей рекуррентной формулы:

$$
\Phi_{\ell}\left(\omega_{1}, \omega_{2}, \ldots, \omega_{\ell}\right)=\Phi_{1}\left(\omega_{1}\right) \Phi_{\ell-1}\left(\omega_{2}, \ldots, \omega_{\ell}\right)-\sum_{j=2}^{\ell} \Phi_{\ell-1}\left(\omega_{2}, \ldots, \omega_{1}+\omega_{j}, \ldots, \omega_{\ell}\right) .
$$

Свойство 2. Последовательность $\left\{\Phi_{\ell}\right\}$, соответствующая $g$, обрывается на $(n+1)$-м шаге, т.е. $\Phi_{n+1}\left(\omega_{1}, \ldots, \omega_{n+1}\right) \equiv 0$.

2. Пусть $X=\left[\mathbf{x}_{1}, \mathbf{x}_{2}, \ldots, \mathbf{x}_{n}\right]-$ набор точек из $\mathbb{C}^{m}$.

Введем отображение $f_{X}: \mathbb{C}^{m} \rightarrow \operatorname{Sym}^{n}(\mathbb{C})$ по формуле

$$
f_{X}(\mathbf{z})=\left[\left\langle\mathbf{z}, \mathbf{x}_{1}\right\rangle, \ldots,\left\langle\mathbf{z}, \mathbf{x}_{n}\right\rangle\right]
$$

Теорема 1. Отображение $g$ имеет вид $f_{X}$ для некоторого набора $X$ тогда и только тогда, когда g обладает свойствами 1 и 2.

Этот резултат дает нетривиальное обобщение “аналога теоремы Пэли-Винера для комплексного преобразования Радона" (в терминологии [3]) на случай конечных мер.

3. Пусть

$$
A(t, \mathbf{z})=t^{n}-a_{1}(\mathbf{z}) t^{n-1}+\cdots+(-1)^{n} a_{n}(\mathbf{z}),
$$

где $a_{k}(\mathbf{z})$ - формы степени $k$. Корни $\left[t_{1}, \ldots, t_{n}\right]$ уравнения $A(t, \mathbf{z})=0$ определяют отображение $f_{A}: \mathbb{C}^{m} \rightarrow \operatorname{Sym}^{n}(\mathbb{C})$. 
СледСтВИЕ 1. Отображение $f_{A}$ имеет вид $f_{X}$, m.e. $\left[t_{1}, \ldots, t_{n}\right]=\left[\left\langle\mathbf{z}, \mathbf{x}_{1}\right\rangle, \ldots,\left\langle\mathbf{z}, \mathbf{x}_{n}\right\rangle\right]$ $u$, следовательно, $A(t, \mathbf{z})=\prod_{i=1}^{n}\left(t-\left\langle\mathbf{z}, \mathbf{x}_{i}\right)\right\rangle$, тогда и только тогда, когда $f_{A}$ обладает свойством 2.

Таким образом, получен алгоритм проверки на разложимость полинома $A(t, \mathbf{z})$ в произведение линейных множителей. Из соотношения

$$
\frac{d}{d t} \log A(t, \mathbf{z})=\sum_{k \geqslant 0} t^{-k-1} s_{k} \circ f_{A}(\mathbf{z})=\sum_{k \geqslant 0} t^{-k-1} \sum_{|\omega|=k}\left(\begin{array}{l}
k \\
\omega
\end{array}\right) \alpha_{\omega} \mathbf{z}^{\omega}
$$

находим значения параметров $\alpha \omega$, что определяет отображение $\Phi$. Требуется проверить условие $\Phi_{n+1} \equiv 0$. Используем следующее явное выражение [1]:

$$
\Phi_{\ell}\left(\omega_{1}, \ldots, \omega_{\ell}\right)=\sum_{\sigma} \operatorname{sign}(\sigma) \Phi_{\sigma}\left(\omega_{1}, \ldots, \omega_{\ell}\right),
$$

где $\sigma$ пробегает группу перестановок $S_{\ell}$. Разлагая $\sigma$ в произведение циклов $\gamma_{1} \cdot \gamma_{2} \cdots \gamma_{q}$, задаем $\Phi_{\sigma}=\Phi_{\gamma_{1}} \Phi_{\gamma_{2}} \ldots \Phi_{\gamma_{q}}$, и для $\gamma=\left(i_{1}, \ldots, i_{r}\right) \subset(1, \ldots, \ell)$ полагаем $\Phi_{\gamma}\left(\omega_{1}, \ldots, \omega_{\ell}\right)=$ $\Phi\left(\omega_{i_{1}}+\cdots+\omega_{i_{r}}\right)$.

4. Пусть, как и выше, $a_{n}(\mathbf{z})$ - ненулевая форма степени $n$. Выберем точку $\mathbf{y} \in \mathbb{C}^{m}$ такую, что $a_{n}(\mathbf{y})=1$, и положим $A_{\mathbf{y}}(t, \mathbf{z})=a_{n}(\mathbf{z}+t \mathbf{y})$. Тогда

$$
A_{\mathbf{y}}(t, \mathbf{z})=t^{n}+\sum_{k=1}^{n}(-1)^{k} a_{n}(\mathbf{z}, \mathbf{y}) t^{n-k}
$$

Согласно следствию 1 полином $A_{\mathbf{y}}(t, \mathbf{z})$ разлагается в произведение линейных множителей (и, следовательно, форма $a_{n}(\mathbf{z})$ имеет вид $\left.\prod_{i=1}^{n}\left\langle\mathbf{z}, \mathbf{x}_{i}\right\rangle\right)$ тогда и толко тогда, когда $f_{A_{\mathbf{y}}}$ обладает свойством 2. Таким образом, описанный выше алгоритм применим к решению задачи о разложении форм на линейные множители (см. [4]).

5. Рассмотрим алгебру Ли $W_{m}$ формалшных векторных полей на $\mathbb{C}^{m}$. Базис в $W_{m}$ составляют поля $D_{\omega, j}=\mathbf{z}^{\omega} \frac{\partial}{\partial z_{j}}$.

Теорема 2. Алгебра Ли $W_{m}$ действует на функииях $\Phi_{\ell}$ по формуле

$$
D_{\omega, j} \Phi_{\ell}\left(\omega_{1}, \omega_{2}, \ldots, \omega_{\ell}\right)=\sum_{k=1}^{n}\left\langle\omega_{k}, e_{j}\right\rangle \Phi_{\ell}\left(\omega_{1}, \omega_{2}, \ldots, \omega_{k}+\omega-e_{j}, \ldots, \omega_{\ell}\right),
$$

где $e_{j}-j$-й орт.

СлеДСтвиЕ 2. Для проверки свойства 2 достаточно ограничиться вычислением значений $\Phi_{n+1}$ только на представителях орбит действия обертывающей алгебры алгебры Ли $W_{m}$.

\section{СПИСОК ЛИТЕРАТУРЫ}

[1] В. М. Бухштабер, Е. Г. Рис // УМН. 1997. Т. 52. № 2. С. 159-160. [2] V. M. Buchstaber, E. G. Rees // uk.arXiv.org: math.CO/0109122. [3] И. М. Гельфанд, М. И. Граев, Н.Я. Виленкин. Интегралшная геометрия и связанные с ней вопросы теории представлений. М.: Физматгиз, 1962. (Обобщенные функции, вып. 5.) [4] I. M. Gelfand, M. M. Kapranov, A. V. Zelevinsky. Discriminants, Resultants, and Multidimensional Determinants. Boston: Birkhäuser, 1994.

Математический институт им. В. А. Стеклова РАН; 